REDES- Revista hispana para el análisis de redes sociales

Vol. 7,\#5, Oct./Nov. 2004.

http://revista-redes.rediris.es

\title{
Apoyo a la Unión Europea y difusión de una identidad europea a través de las redes personales
}

\author{
Livia García Faroldi, Universidad de Málaga, España
}

\begin{abstract}
This research tests the hypothesis that attitudes towards the European Union as well as the degree of identification to the EU tends to be reinforced or weakened within personal networks. European identity is considered as an innovation that spreads (or not) through personal networks. This innovation is considered as socially prestigious and, because of that, it is adopted in first place by those who are opinion leaders in their network. Using Merton's distinction between "locals" and "cosmopolitans" leaders, it is supposed that these influents have a more cosmopolitan profile than the other members of their network. Indeed, it is assumed that people who are more familiar with the European project exert an important influence in their network because they are considered "experts". Finally, it is tested if people who occupy bridge positions between local and cosmopolitan parts of the network promote the diffusion of information about European Union (attitudes, opinions and an identity) towards weak ties.
\end{abstract}

Key Words: attitudes, personal networks, diffusion of information, European Union.

\section{RESUMEN}

La investigación parte de la hipótesis de que el desarrollo de actitudes hacia la Unión Europea y la identificación con ésta tiende a ser reforzada o debilitada dentro de las redes personales de las que forman parte los individuos. Se considera que la identidad europea es una innovación que se difunde - o no- a través de las redes personales. Esta innovación se considera prestigiosa socialmente y, por ello, es adoptada en primer lugar por quienes ejercen un liderazgo de opinión en la red. Empleando la distinción de Merton entre líderes locales y cosmopolitas, se espera que estos influyentes tengan un perfil más cosmopolita que el resto de miembros de su red. Además, se cree que son aquellos más familiarizados con el proyecto político europeo los que son considerados "expertos" en su red y por ello ejercen un liderazgo. Por último, se quiere comprobar si son las personas que ocupan posiciones de puente entre partes de la red locales y cosmopolitas las que promueven la difusión de información sobre la Unión Europea -actitudes, opiniones $y$, en último término, una identidad- a través de los lazos débiles.

Palabras clave: actitudes, redes personales, difusión de la información, Unión Europea.

\section{La formación de una identidad europea como resultado de}

\section{características personales}


Desde su creación en 1957 con la firma del Tratado de Roma, la Comunidad Económica Europea -rebautizada como Unión Europea en el Tratado de la Unión Europea que entró en vigor en 1992- ha sido objeto de estudio desde diversas disciplinas sociales. El aspecto sobre el que centra la atención el presente texto es cómo se desarrollan las actitudes positivas o negativas hacia la UE y qué relación existe entre dichas actitudes y la identificación con la Unión.

Varios enfoques han intentado explicar las actitudes a favor y en contra de la Unión Europea recurriendo a variables atributivas y, aunque son menos numerosos o se han ocupado de ello de manera tangencial, también algunos autores han sugerido factores individuales que pueden influir en que una persona desarrolle una identidad europea. [1] En este apartado se realiza una revisión de los análisis que más relevancia han tenido en este campo. El enfoque cosmopolita es uno de los más conocidos y empleados al estudiar las actitudes hacia la Unión y el apoyo a las políticas de integración europea. Dentro de éste, se puede diferenciar una primera vertiente, defendida por Inglehart, que se centra en la hipótesis de que el apoyo a la UE se relaciona con el desarrollo de una visión postmaterialista; en segundo lugar, el análisis de Deutsch y sus seguidores recurre a variables estructurales para explicar el grado de cosmopolitismo de un individuo. Otra perspectiva muy conocida es la que analiza al individuo como un actor racional que hace un balance de los beneficios y perjuicios del proyecto europeo y es dicho balance el que explica el desarrollo de actitudes a favor o en contra de la Unión. Dentro de este enfoque, cabe distinguir varias vertientes, aunque en este texto se explicará únicamente la perspectiva utilitarista desarrollada por Gabel y Palmer. ${ }^{[2]}$

\section{El enfoque cosmopolita}

La conocida teoría de la "revolución silenciosa" de Inglehart ha sido utilizada por el propio autor y por otros estudiosos para analizar el apoyo a la integración europea por parte de los ciudadanos de los Estados miembros. ${ }^{[3]}$ Según este enfoque, dos variables influyen en la formación de las actitudes: las orientaciones de los valores políticos y el nivel de habilidades políticas movilización cognitiva-. ${ }^{[4]} \mathrm{El}$ autor distingue entre dos orientaciones, 
materialista y postmaterialista, y afirma que la integración europea encaja mejor con la orientación postmaterialista porque 1) los materialistas son menos cosmopolitas, tienen una mente más estrecha que los postmaterialistas y 2) los postmaterialistas están más deseosos de llenar una cierta necesidad de pertenencia, una necesidad que puede satisfacerse mejor identificándose con la idea de Europa antes que con la de Estadonación, que es demasiado materialista para ellos.

En cuanto a la movilización cognitiva, las habilidades políticas determinan si una persona es capaz de procesar información de un cierto nivel de abstracción, como es el caso de la información sobre política internacional y la integración europea. Inglehart utiliza el concepto "abstracción" para indicar la facilidad con que las personas entienden los mensajes políticos y los relacionan con sus propios pensamientos y situación personal. En la mayoría de los casos, la distancia de la fuente de información es un buen indicador del nivel de abstracción. El sociólogo norteamericano espera que las personas con más habilidades políticas vean la Unión Europea como más familiar y menos amenazante que la gente con menos habilidades. ${ }^{[5]}$

Los modelos que se basan en los trabajos de Deutsch ${ }^{[6]}$ hacen hincapié en el papel que tienen variables estructurales como la frecuencia de viajes al extranjero para desarrollar una visión cosmopolita. Los resultados de una encuesta realizada por el CIS en 1988 mostraron que la cercanía afectiva se relaciona positivamente con la cercanía geográfica y con la percepción de que estos pueblos "tienen cosas en común con España", pero, aunque estos sentimientos se distribuyen por igual en toda la población, se pueden encontrar diferencias en el grado en que se siente más o menos cercanos a los europeos en general: "lo que determina un mayor grado de cercanía a todos los países europeos es el haber salido de España". ${ }^{[7]}$

En este estudio dirigido por Moral se incluyeron preguntas para averiguar la confianza que despiertan los socios europeos: predomina claramente dicha confianza, sobre todo hacia Italia, los países del Benelux y Dinamarca, situándose en la cola los irlandeses e ingleses (incluso predomina ligeramente la desconfianza en estos últimos). Pese a todo, se ha producido un aumento de la confianza en todos los países miembros desde que 
ingresó España, por lo que se podría interpretar, como hace Moral, que "el ingreso ha llevado a los españoles a un proceso de identificación con el resto de los europeos que ha provocado una radical reducción de las posibles desconfianzas que, con anterioridad al ingreso, podrían despertar". [8] También ha aumentado la confianza que los países del Norte muestran hacia los del Sur, mejorando la opinión acerca de los socios mediterráneos aunque aún no se llega a los niveles de confianza que se tiene en los países nórdicos. Por lo tanto, la confianza surge a posteriori, después de la asociación. ${ }^{[9]}$ En un estudio posterior de Díez Medrano, ${ }^{[10]}$ se incluyeron varias preguntas para medir la evaluación que se realiza de ciudadanos de otros países de la Unión. Su análisis de regresión muestra que los que sienten más afinidad por los habitantes de otros países de la UE se identifican más con la Unión.

Por otro lado, las investigaciones de De Federico ${ }^{[11]}$ y Schmidberger ${ }^{[12]}$ concluyen que las redes de amistad y la comunicación frecuente con otros miembros de la Unión puede llegar a modificar la percepción de las fronteras nacionales -al menos en la esfera privada- y aumentar el grado de identidad europea. Schmidberger compara las identificaciones nacionalistas en las zonas fronterizas de Francia y Alemania con las del resto del país. En esta situación, señala el autor, podría esperarse que las identidades fueran reforzadas, en cambio, lo que se encuentra es una renegociación de las identidades a través de la interacción diaria y de los conflictos, haciendo que las identidades de las personas que viven en la frontera sean más complejas y que la identidad europea resulte más relevante que la de sus connacionales que viven en el interior del país. Por su parte, De Federico concluye que, en general, cuanto más intensas son las redes de amistad, menos es probable es que un amigo sea considerado una persona extrajera y con más probabilidad la nacionalidad de dicho amigo pase desapercibida. En las relaciones de amistad se produce un movimiento de la frontera de lo que se considera extranjero, debido a la propia dinámica y lógica de la amistad.

\section{El enfoque utilitarista}


Los autores que defienden el enfoque utilitarista consideran, de manera general, que el apoyo a la Unión está condicionado por la percepción de los beneficios que comporta formar parte de ella. Los estudios realizados en España muestran que los ciudadanos tienen una visión utilitarista de la Unión pero, como señala Díez Medrano, los españoles perciben beneficios respecto a temas generales como son el funcionamiento de la democracia, la modernización de la sociedad española, el papel de España en el mundo, pero son mucho más escépticos al valorar el efecto de la pertenencia a la UE sobre temas económicos. Por lo tanto, el apoyo global de los españoles no parece que se base en que el país se está beneficiando económicamente de su pertenencia a la UE. De hecho, el autor señala que no se puede "desdeñar la posibilidad de que, al pensar en los beneficios globales relacionados con la Unión Europea, los entrevistados proyecten actitudes respecto a la Unión Europea que tienen más que ver con su reacción afectiva hacia la Unión Europea que con un cálculo pormenorizado de costes y beneficios".13]

Gabel y Palmer ${ }^{[14]}$ consideran que la liberalización de los mercados, la libertad de circulación de las personas y la liberalización del mercado de trabajo afectan de manera diferente a los ciudadanos europeos según su nivel educativo, sus habilidades ocupacionales y su nivel de ingresos, por lo que apoyarán en mayor medida a la Unión Europea aquellos que se beneficien más de estas medidas. Además, la libertad de circulación de materias y personas influye diferenciadamente según el lugar de residencia, siendo los principales beneficiados los habitantes de zonas fronterizas, y por tanto el apoyo será mayor entre éstos. Estos autores y Anderson y Reichtert $\frac{[15]}{[1}$ han realizado análisis multivariables que corroboran estas hipótesis, sin embargo, los propios autores reconocen que no son evidencias concluyentes porque el análisis no controla explicaciones alternativas. En un

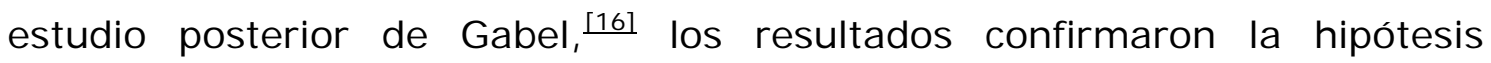
utilitarista: los encuestados con menos educación apoyan menos el proceso que los de mayor educación, al igual que los que tienen mayores habilidades ocupacionales (profesionales y ejecutivos) frente a los que tienen menos (trabajadores manuales y desempleados). Asimismo, el apoyo también se correlaciona positivamente con el nivel de ingresos. Por otro 
lado, el lugar de residencia parece tener alguna relación positiva con el apoyo, pero es una evidencia no muy intensa.

\section{El estudio de la identidad europea como el proceso de difusión de una innovación}

En la investigación que se realiza -que será explicada en el último epígrafese considera que la identidad europea se difunde a diferentes ritmos entre los miembros de la red. Al analizar la difusión se suele diferenciar entre innovadores, adoptadores tempranos, mayoría temprana, mayoría tardía y rezagados. En la mayoría de las ocasiones existen líderes de opinión que dan información y consejo sobre la innovación y la adoptan antes que sus seguidores. El liderazgo de opinión es el grado en que un individuo es capaz de influenciar las actitudes o el comportamiento manifiesto de otros individuos informalmente en un modo deseado con una relativa frecuencia. Es un tipo de liderazgo informal más que una función de una posición formal. $Y$ es ganado y mantenido gracias a la competencia técnica, la accesibilidad social y la conformidad con el sistema normativo. ${ }^{[17]}$

Sin embargo, hay que tener en cuenta el sistema social para averiguar si los líderes informales serán más innovadores que el resto de los miembros o no. El estudio realizado por Becker en este campo concluyó que aquellos dentro de la red que adoptan la innovación - los innovadores- pueden ejercer el papel de líderes de opinión de dicha red si la innovación se considera ventajosa y prestigiada socialmente; en caso contrario, si la innovación se percibe como arriesgada, será adoptada antes por los miembros marginales. $\stackrel{[18]}{ }$

Los líderes de opinión suelen tener un mayor nivel socioeconómico y educativo que el resto de los miembros de la red, consumen más medios de comunicación, están más en contacto con agentes de cambio -lo cual conlleva un mayor grado de cosmopolitismo- y son accesibles, es decir, mantienen redes interpersonales con sus seguidores a través su participación social en distintas organizaciones. ${ }^{[19]}$

Varios de estos rasgos - nivel educativo, nivel económico y participación social- ya fueron incluidos por Galtung en los años sesenta al construir un 
índice para situar a las personas en posiciones dentro de la estructura social. ${ }^{[20]}$ El autor divide la sociedad en tres partes: un núcleo de decisores (decision-making nucleus), el centro social y la periferia social: dejando de lado a la minoría que toma las decisiones, Galtung define el centro social como aquél que ocupa las posiciones que están socialmente recompensadas, y la periferia social la que ocupa las posiciones menos

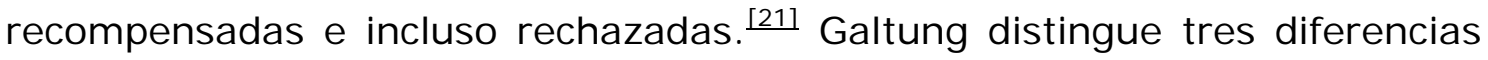
estructurales entre el centro y la periferia: el centro social tiene altos índices de participación social (medidos a través del consumo de medios y su pertenencia a asociaciones), y tiene altos niveles de conocimientos sobre asuntos políticos y de mantenimiento de opiniones sobre esta materia. Frente a ellos, la periferia social tiene una baja participación, pocos conocimientos y no tiene opiniones bien definidas y sólidas. Galtung muestra un enfoque estructural al señalar que estas características estructurales de los individuos tienen implicaciones para sus actitudes, aunque la diferencia no se basa tanto en las opiniones que se mantienen como en el modo en que esas opiniones son mantenidas.

La hipótesis del autor es que, como el centro es quien accede a los medios de comunicación y además tiene algo que comunicar, el proceso de comunicación se emite desde el centro hacia la periferia. En cuanto a la recepción, aunque gran parte del proceso de comunicación sobre asuntos políticos se da dentro del centro, las personas de la periferia están ligadas a este proceso a través de contactos informales con personas del centro.

Como ya se comentó anteriormente, dentro de la red personal del individuo existen personas que ocupan posiciones de liderazgo informal y ejercen una influencia sobre el resto de los miembros de la red. Lazarsfeld, Berelson y Gaudet fueron quienes descubrieron, en los años cuarenta, la existencia de estos líderes de opinión "horizontales" que influyen en su entorno inmediato. [22]. Durante la campaña presidencial estadounidense de 1940, los autores investigaron los efectos de la prensa y la radio en la decisión del voto en una comunidad de Ohio. Los datos mostraron que los efectos de los medios de comunicación eran pequeños en comparación con el papel jugado por las influencias personales. Concluyeron que los votantes se deciden 
ajustándose al clima político de su entorno social. El hallazgo fue relevante ya que, hasta entonces, se había asumido que las opiniones se formaban en la elite de la comunidad y descendían hacia el resto de los estratos sociales, se producía un liderazgo "vertical" de la cúspide hacia la base. Esta investigación mostró que existía también un liderazgo de opinión "horizontal" pues, como los propios autores comentaban, estos líderes de opinión "no son idénticos a los individuos socialmente prominentes en la comunidad, ni a los individuos más ricos, ni a los líderes cívicos". [23]

Al igual que Galtung, estos investigadores mostraron su interés en la estructura social al señalar que, al margen y por encima de estos líderes "horizontales", existen una serie de interacciones recíprocas entre los componentes del grupo, que refuerzan las actitudes todavía no concretadas de cada persona. Sobre la base de estas interacciones, se cristaliza la distribución de opiniones y actitudes articuladas. Los efectos de los medios de comunicación, que eran el foco de interés de estos estudiosos, sólo pueden comprenderse teniendo en cuenta estas relaciones entre los destinatarios de la comunicación.

También fue a lo largo de este estudio cuando se formuló la hipótesis de que existía un flujo en dos pasos en la comunicación (two-step flow of communication) entre los medios de masas y el público: en cada estrato social, los líderes de opinión tenían una mayor probabilidad de exponerse a los medios y éstos, a su vez, transmitían esta información al resto de las personas. Las ideas, por tanto, fluyen de la radio y la prensa hasta los líderes de opinión y de éstos a los sectores menos activos de la población. Estos líderes son definidos por los autores como aquellos individuos que se caracterizan por estar muy implicados e interesados por el tema y dotados de más conocimientos sobre el mismo, que actúan como mediadores entre los medios de comunicación y el resto de los individuos, que están menos interesados o tienen una menor implicación en dicho asunto. ${ }^{[24]}$

\section{Relaciones cosmopolitas y posiciones de puente dentro de la red}

Ya se ha comentado anteriormente que se ha comprobado que existe una relación entre el haber viajado a otros países de la Unión Europea y el grado 
de cercanía hacia los ciudadanos de estos territorios. Siguiendo a Deutsch, se puede suponer que estos viajes y también las estancias en otros países influyen positivamente en el grado de cosmopolitismo de una persona. Por ello, resulta interesante investigar estos dos fenómenos y, además, tener en cuenta si existe contacto con personas de otros países comunitarios, el grado de intimidad de dichas relaciones en el caso de que existan y si estos individuos viven en su país de origen o en España, ya que todos estos factores pueden incidir en el grado de localismo/cosmopolitismo de la red personal del sujeto.

En este sentido, el modelo de análisis de las redes personales elaborado por Ferrand, ${ }^{[25]}$ se propone considerar la potencialidad que tiene cada red para articular las sub-redes locales y no locales, es decir, si conectan muchos o pocos lazos locales a muchos o pocos lazos no locales. El autor identifica cuatro tipos de micro-estructuras, una local, una no local y dos local/no local, ya que se pueden distinguir, en términos del autor, los "difusores", que son estructuras en que la mayoría de las relaciones son locales y los "cosmopolitas",estructuras compuestas por un número igual o mayor de no locales que de locales. Ambos tipos conectan la vida social local con los ámbitos externos: el primero es probablemente más capaz de difundir localmente los recursos externos y la información, dado que la mayor parte de sus relaciones son locales, mientras que el segundo probablemente es más capaz de capturar recursos más variados en la red externa, dado que la mayoría de sus relaciones no son locales.

Para los intereses de la presente investigación, se ha preferido utilizar una escala distinta a la de Ferrand, que distingue únicamente entre lazos de la localidad con lazos extra-locales. En vez de ello, se ha preferido emplear la escala creada por Requena, que distingue tres categorías dentro de los lazos locales y dos categorías de extra-locales, ampliando las categorías que distinguen a los segundos. ${ }^{[26]}$

Esta reflexión sobre las redes "mixtas" (local/no local) enlaza con una segunda característica importante en el análisis de la red: las posiciones de puente. Normalmente, la comunicación dentro de la red ocurre entre individuos homogéneos: la mayoría de los individuos se sienten cómodos 
interactuando con personas que son bastante similares a ellos. La heterogeneidad, en cambio, puede causar disonancia cognitiva porque un sujeto es expuesto a mensajes que son inconsistentes con sus creencias, causando un estado psicológico incómodo. La homogeneidad y la comunicación efectiva se generan mutuamente: cuanta más comunicación se produce entre dos miembros de una díada, más probable es que se conviertan en homogéneos, y cuanto más homogéneos son, más probable es que su comunicación sea efectiva. Sin embargo, la comunicación heterogénea tiene un potencial especial informacional, aunque sólo se lleve a cabo raramente: los lazos heterogéneos de una red a menudo conectan a dos apiñamientos y estos lazos interpersonales son especialmente importantes para transmitir información sobre innovaciones.

Es más, la homogeneidad puede actuar como una barrera invisible para el flujo de innovaciones dentro de un sistema: las nuevas ideas normalmente entran en el sistema a través de los miembros más innovadores y de los estatus más altos. Un alto grado de homogeneidad significa que estos individuos de las elites interactúan sólo con miembros de su elite, y por tanto la innovación no se difunde entre la no-elite: las ideas se difunden horizontalmente, no verticalmente, dentro del sistema. Cuando existe un cierto grado de heterogeneidad, existen una serie de características específicas de los líderes y los seguidores que ya se han comentado en líneas anteriores: los líderes suelen tener un estatus socioeconómico más alto, mayor nivel educativo, se exponen más a los medios de comunicación, son más cosmopolitas y más innovadores. [27]

Cuando se produce una comunicación heterogénea frecuentemente ocurre entre personas que están unidas por lazos débiles. Granovetter ${ }^{[28]}$ destaca la importancia que tienen estos vínculos débiles como puentes, entendiendo por puente el único camino entre dos puntos. Un vínculo fuerte difícilmente es un puente, por lo que suele suceder que, aunque no todos los lazos débiles son puentes, sí ocurre que todos los puentes son lazos débiles. En redes amplias es difícil que un vínculo dado sea el único camino entre dos puntos y se le puede considerar puente, pero sí se puede contemplar esta función en redes más pequeñas, analizando los puentes locales. La 
conclusión que se extrae de todo ello es que cualquier cosa que quiera ser difundida puede llegar a un gran número de personas y atravesar una gran distancia social cuando se emplean vínculos débiles antes que fuertes.

Basándose en las reflexiones teóricas de Granovetter, el análisis de los huecos estructurales (structural holes) desarrollado por Burt sugiere que las posiciones de puente otorgan a quienes las ocupan recursos informativos, de poder e influencia mayores que otras posiciones. ${ }^{[29]}$ La idea principal del argumento de los agujeros estructurales es que el capital social se crea gracias a que el individuo controla la difusión de la información entre distintas partes de la red. Los vacíos que existen en la estructura social crean una ventaja competitiva para los individuos cuyas relaciones cruzan estos huecos. Los miembros de cada lado del vacío estructural circulan por diferentes flujos de información y, por ello, las personas que actúan como puentes tienen la oportunidad de comunicar ambos flujos de información.

\section{I ntegración de aspectos atributivos y relacionales en el análisis de la identificación con la Unión Europea}

El objeto de estudio de la investigación que se realiza es doble: por un lado, averiguar qué actitudes existen en la población española hacia la Unión Europea y su proceso de integración y cuáles son las variables más relevantes en el desarrollo de dichas actitudes; por otro, descubrir la relación que existe entre dichas actitudes y la identificación con la Unión.

En el estudio se han incluido variables atributivas y reticulares para explicar cómo se desarrollan las actitudes hacia la Unión Europea y qué factores influyen en el desarrollo de una identificación con ésta. Los factores individuales que se incorporan son aquéllos que han destacado los enfoques cosmopolita y utilitarista, mencionados en el primer epígrafe. En primer lugar, se incluyen ítems para averiguar el grado de cosmopolitismo de los sujetos, definiendo el cosmopolitismo tanto desde la perspectiva de Inglehart como la de Deutsch. Por un lado, se realizan preguntas para detectar valores postmaterialistas y materialistas así como el nivel de habilidades políticas. Por otro, se quiere saber si se han realizado viajes al extranjero, los motivos para ello y el tiempo de estancia. En segundo lugar, se incluyen varias preguntas para corroborar la hipótesis utilitarista: se 
interroga al entrevistado sobre si percibe beneficios para el país y beneficios personales en la pertenencia de España a la Unión Europea y -si los hubiera- qué tipo de beneficios son. También se pregunta por cuáles son los rasgos que mejor definen a la Unión Europea -algunos de ellos con un marcado carácter económico- y también se recogen la ocupación y el nivel de ingresos del sujeto. $[30]$

Estos factores personales se complementan con datos relacionales. Como ya se dijo anteriormente, en la presente investigación se considera la identificación con la UE como una innovación que se difunde a distintos ritmos dentro de la red. Por lo que respecta a si se la debe calificar de una innovación prestigiosa o arriesgada, se supone que es una innovación ventajosa ya que, al menos desde las instituciones políticas de los países implicados, se apoya explícitamente la extensión y consolidación de esta identidad, desarrollando incluso políticas comunitarias que incluyen como uno de sus fines esta identidad. $\stackrel{[31]}{ }$ Por tanto, es presumible que serán las personas que ejercen un liderazgo dentro de su red las que están adoptando primero esta identificación con la Unión. De hecho, las investigaciones realizadas muestran que quienes se identifican en mayor medida con Europa - no sólo en España, sino en otros países miembros- son aquellos con un mayor nivel educativo y socioeconómico. Por tanto, se puede concluir que, más que tratarse de individuos aislados y marginales, son sujetos bien situados dentro de su red social. ${ }^{[32]}$

Además, las investigaciones muestran que los líderes de opinión suelen tener un mayor nivel socioeconómico y educativo que el resto de los miembros de la red, consumen más medios de comunicación, están más en contacto con agentes de cambio - lo cual conlleva un mayor grado de cosmopolitismo- y son accesibles, es decir, mantienen redes interpersonales con sus seguidores a través su participación social en distintas organizaciones. ${ }^{[33]}$ Muchas de estas características están presentes en el índice creado por Galtung, por ello, dicho índice es empleado en la presente investigación para corroborar la hipótesis de que son las personas que ocupan posiciones centrales dentro de su red-definiendo las posiciones a 
través de dicho índice- las que adoptan primero una identidad europea que, progresivamente, se va extendiendo hacia las posiciones más periféricas.

También se pretenden comprobar los rasgos que encontraron Lazarsfeld et al en estos "líderes horizontales", es decir, averiguar si se trata de personas que tienen un mayor interés y una mayor implicación en los asuntos europeos que el resto de miembros de la red. La distinción de Merton entre líderes locales y cosmopolitas resulta útil a este fin, pues se espera que las personas que ejercen una mayor influencia en los temas relacionados con la Unión Europea tengan un perfil más cosmopolita que sus compañeros, mostrando un mayor interés e implicación en los asuntos que trascienden el ámbito local. [34]

Además, como ya se ha visto, se ha descubierto que existe una relación entre el haber viajado a otros países de la Unión Europea y el grado de cercanía hacia los ciudadanos de estos territorios. Es más, se ha verificado el vínculo que existe entre sentir una afinidad por los habitantes de la UE y la identificación con ésta. Es por ello que se incluyen variables que miden el grado de cosmopolitismo de la persona y de su red. Pero existe otro motivo más por el que interesa averiguar hasta qué punto el individuo mantiene contactos con otros países de la Unión se debe a que las investigaciones que han estudiado la difusión de innovaciones han mostrado que las personas buscan información o asesoramiento en las personas que consideran "expertas" en el tema. En el caso de la Unión Europea, se puede suponer que aquellas personas más familiarizadas con el proyecto político y/o que tengan más contactos con el exterior serán consideradas por el resto de los miembros de la red como los "expertos" y, por tanto, tendrán influencia en ellos. $Y$, dado que el mayor contacto con otros países y el desarrollo de lazos de amistad se relaciona positivamente con la identificación con el proyecto europeo, dicha influencia debería orientarse en favor del desarrollo de una identidad europea.

Por último, interesa comprobar si, como la teoría de los lazos débiles sugiere, la difusión de una identidad europea se logra extender a toda la red a través de miembros que ocupan posiciones de puente. Utilizando los conceptos de Ferrand mencionados anteriormente, se puede suponer que 
son los miembros "difusores" y "cosmopolitas" los que pueden hacer circular información sobre la Unión, actitudes, opiniones y, en último término, una identidad europea. Ocuparían así posiciones de puente -y, por tanto, de poder- entre distintas partes de la red, facilitando el flujo de todo tipo de información sobre la Unión a los miembros de la red cuyos contactos son exclusiva o mayoritariamente locales, para los que la Unión Europea significa una referencia lejana y casi desconocida, con la que resulta por tanto muy improbable identificarse.

La utilización conjunta de variables atributivas y reticulares podrá mejorar la comprensión de un fenómeno complejo como es el de la identificación con la Unión. Numerosas investigaciones han mostrado que el apoyo a la Unión y el desarrollo de una identidad europea se relacionan positivamente con el nivel educativo, la posición socioeconómica, el nivel de habilidades políticas, el desarrollo de una visión postmaterialista, entre otros aspectos. Sin negar la relevancia de estos factores, se considera que resultaría más fructífero ponerlos en relación con aspectos reticulares: es probable, por ejemplo, que el nivel educativo y el desempeño de ciertas profesiones se asocie con una mayor frecuencia de viajes al extranjero y de contacto con personas de otros países comunitarios, y ello, a su vez, está vinculado con la cercanía a ciudadanos de otros países comunitarios, y el sentimiento de proximidad a estos ciudadanos con la identificación con la Unión. También podemos suponer que una persona, independientemente de su nivel educativo o socioeconómico, será más proclive a apoyar el proyecto de integración europea e incluso a identificarse con la Unión si en su red éstas son las actitudes mayoritarias; asimismo, un sujeto con alto nivel educativo y buena posición socioeconómica será menos proclive a desarrollar una identidad europea si todos o la mayoría de sus lazos son locales y no tiene contacto con el exterior. Por tanto, se puede concluir que la inclusión de ambos tipos de datos, relacionales e individuales, aumenta el poder explicativo del análisis de cómo y por qué se desarrolla una identificación con la Unión. 
[1] El término "identificación" expresa mejor que el de "identidad" el carácter dinámico del fenómeno de las identidades nacionales -también la europea- que son resultado de un proceso histórico, por lo que preferimos utilizar el primero al segundo, aunque a lo largo de este trabajo se emplearán los dos indistintamente. Asimismo, se prefiere utilizar el concepto "una identidad europea" en vez de "la identidad europea" porque probablemente dicha identidad no tenga un carácter único e inmutable, sino que se defina de distintas formas y se sienta de manera diferente en distintos contextos nacionales y sociales.

[2] Díez Medrano, J. (La opinión pública española y la integración europea: 1994; CIS, Serie Opiniones y Actitudes, Madrid, 1994) realiza una revisión de estos y otros enfoques e incluye ítems para contrastar la validez de cada uno de ellos en su propia investigación.

[3] Inglehart, R.; "An End to European Integration?", in American Political Science Review; 61, 1967; "Public opinion and regional integration", in International Organization,;24, 1970; "Cognitive Mobilization and European Identity", in Comparative Politics; 3, 1970; "Changing Value Priorities and European Integration", in Journal of Common Market Studies; 10, 1971; "Long Term Trends in Mass Support for European Unification; in Government and Opposition; 12, 1977; The silent revolution: Changing Values and Political Styles among Western Publics; Princeton University Press, Nwe Jersey, 1977; Cultural Shift in Advanced Industrial Society; Princeton Universtiy Press, New Jersey, 1990.

[4] Inglehart observa tres tipos de consecuencias de la "revolución silenciosa", a nivel individual, nivel meso y nivel macro, aunque en este artículo sólo se comenta el primero de estos niveles.

[5] Para una crítica de la perspectiva de Inglehart y una contrastación de sus hipótesis, cfr. Janssen, J. I. $\mathrm{H}$.; "Postmaterialism, Cognitive Mobilization and Publlic Support for European Integration", en British Journal of Political Science; Cambridge University Press, vol. 21, 1991.La importancia de la movilización cognitiva como variable explicativa del apoyo a la integración europea se ha confirmado en investigaciones españolas (Díez Medrano J.; op. cit.).

${ }^{[6]}$ Deutsch, K. W.; Nationalism and Social Communication; MIT Press, Cambridge, 1952.

[7] Moral, F.; La opinión pública española ante Europa y los europeos; CIS, Serie Estudios y Encuestas, $n^{\circ}$ 17, Madrid, 1989, p. 23.

${ }^{[8]}$ Moral, op. cit., p. 35.

[9] Una posición similar defendía Granovetter al señalar que la confianza no tiene por qué surgir antes en el tiempo que la asociación, sino que podía nacer a raíz de esta última ("Economic action and social structure: The problem of embeddedness", American Journal of Sociology, 91, 1985, pp. 481-510).

[10] Díez Medrano, J.; op. cit.

[11] De Federico, A.; "Networks and Identities. Borders of solidarity and borders of identification of European exchange students", presented at the Conference "Networks and Transformations", organized by the Global Studies Association (GSA) at Manchester Metro politan University, Manchester, UK, July 2001.

[12] Schmidberger, M.; "EU-Akzeptanz und europäische Identität im deutsch-französischen Grenzgebiet", en Aus Politizk und Zeitgeschichte; B 25-6, pp. 18-25.

[13] Díez Medrano, op. cit., p. 50. 
[14] Gabel, M. y Palmer, H.; "Understanding Variation in Public Support for European Integration"; European Journal of Political Research, n² 27, 1995, pp. 3-19.

${ }^{[15]}$ Anderson, C. y Reichtert, M. S.; "Economic benefits and Support for Membership in the EU"; Journal of Public Policiy; nº 15, 1996.

[16] Gabel, M.; "Public Support for European Integration: An Empirical Test of Five Theories", en The Journal of Politics; vol. 60, nº 2, 1998.

${ }^{[17]}$ Rogers, E. M.; Diffusion of innovations; Macmillan, New York, 1983, cap. 1.

${ }^{[18]}$ Becker, M. H.; "Sociometric Location and Innovativeness: Reformulation and Extension of the Diffusion Model", en American Sociological Review, nº 35, 1970, pp. 267-282.

[19] Rogers, E.M., (Diffusion of innovations; Macmillan, New York, 1983) realiza una revisión de los estudios con un enfoque reticular que han analizado la difusión de innovaciones y dedica un capítulo a los líderes de opinión.

[20] Galtung, J.; "Foreign policy opinion as a function of social position", en Journal of Peace Research, 3-4, 1964, pp. 206-231.

[21] La escala de Galtung puntúa del 0 al 8 a las personas, otorgando un punto por cada ítem recompensado socialmente que la persona ocupa (centro social) y un 0 si ocupa la posición no recompensada (periferia social). Así, puntúa como 1 ser varón, tener entre 30 y 59 años, tener estudios superiores a los primarios, ingresos por encima de la media, vivir en entorno urbano o suburbano, que su localización geográfica sea central, ser autónomo o trabajador de cuello blanco y trabajar en los sectores secundario y terciario. Las personas que puntúan de 0 a 2 se sitúan en la perifera, de 3 a 5 ocupan posiciones intermedias y de 6 a 8 están en el centro social.

${ }^{[22]}$ Lazarsfeld, P. F., Berelson, B. R. y Gaudet, H.; The People's Choice. How the voter makes up his mind in a presidential campaign; Columbia University Press, Nueva York, 1954.

[23] Op. cit., p. 50 y cap. XVI.

${ }^{[24]}$ Op. cit., p. 151.

[25] Ferrand, A.; "Las comunidades locales como estructuras meso"; Redes, nº 3, 2002.

[26] Requena Santos, F. ("Relaciones de amistad en los barrios de la ciudad de Málaga"; Anuario de la UNED, $\mathrm{n}^{\circ}$ 7, 1993, pp. 215-223). El autor califica a los cosmopolitas como comunitarios. Requena preguntó al entrevistado si los amigos residían 1) en la misma casa o bloque de pisos; 2) en el mismo barrio; 3) en la misma ciudad; 4) en otra ciudad o pueblo de la provincia; 5) fuera de la provincia. El autor califica de locales a aquellos que puntúan de media hasta 3 , y comunitarios a los que puntúan por encima de esa cifra.

[27] Rogers, op. cit., pp. 274-277.

[28] Granovetter, M.; "The Strength of Weak Ties", en American Journal of Sociology; no 78, 1973, pp. 1360-1380.

${ }^{[29]}$ Burt, R.S., "Structural Holes versus Network Closure as Social Capital", en Nan Lin, Karen S. Cook y R.S. Burt (eds.), Social Capital: Theory and Research; Aldine de Gruyter, 2001. 
[30] El lugar de residencia no se puede emplear como variable en esta investigación ya que el trabajo de campo se ha desarrollado en una única provincia española.

[31] La Comisión Europea considera que uno de los fines del programa de intercambio estudiantil SOCRATES es "desarrollar el sentido de pertenencia a una identidad europea, reforzar el espíritu de la ciudadanía europea y reforzar la comprensión y la solidaridad entre los pueblos de la Unión Europea" (Comisión Europea, Education Formation Jeunesse, SOCRATES Vade-mecum, 1995). (Traducción propia).

${ }^{[32]}$ Los marcos de referencia históricos en que se mueve cada país también son relevantes a la hora de analizar el apoyo hacia la Unión Europea. Un estudio de dichos marcos en España, Alemania y Gran Bretaña ha sido realizado por Díez Medrano, J.; Framing Europe: Attitudes to European Integration in Germany, Spain, and the United Kingdom, Princeton University Press, 2003.

[33] Rogers, E.M., (Diffusion of innovations; Macmillan, Nueva York, 1983) realiza una revisión de los estudios con un enfoque reticular que han analizado la difusión de innovaciones y dedica un capítulo a los líderes de opinión.

${ }^{[34]}$ Merton, R.K.; "Patterns of influence. A study of interpersonal influence and of communications behavior in a local community", in Lazarsfeld and Stanton (eds.), Communication research 1948-1949, Arno Press, New York, 1949. 